\title{
AVALIAÇÃO DO ESTRESSE DE IDOSOS PRATICANTES DE DEFESA PESSOAL
}

\author{
Evaluation of stress in elderly practitioners of personal defense
}

Onacir Carneiro Guedes, Rosilene Maria de Lucena Guedes

\section{Resumo}

Inúmeros fatores afetam o modo como o idoso desempenha determinada tarefa, incluindo o estado de saúde, as características psicológicas e as exigências da tarefa. Mesmo com as perdas físicas, alguns idosos conseguem superar traumas e dificuldades, sem sofrerem as conseqüências negativas do estresse. Os idosos, com o objetivo de melhorar seu estresse, praticam tai chichuan, hidroginástica, natação, além de outras atividades relaxantes. Entretanto, pouco se conhece da Defesa Pessoal como alternativa para reduzir o estresse de pessoas idosas. Deste modo, o presente estudo teve como objetivo verificar de que modo a Defesa Pessoal contribui para a diminuição do estresse de idosos. Participaram do estudo 39 voluntários, sendo 24 homens e 15 mulheres, selecionados no Programa de Atividades Físicas do Conselho de Educação Permanente da Universidade Livre de Bruxelas. Os idosos participaram, por um período de 12 meses, de um Curso de Defesa Pessoal, adaptado de acordo com as características físicas dos mesmos. Foi empregado o Teste de Eficácia de Reação ao Estresse Stroop Color-Word Test - de Jensen e Romer ${ }^{(1)}$, para medir as atitudes dos sujeitos em situações de estresse. Este teste é composto dos fatores de Rapidez Geral (RG), Rapidez Específica (RE) e Interferência (INT), tendo sido aplicado no início e no final do curso. O resultado mostrou que as idosas diferenciaram-se estatisticamente dos homens, no pré-teste de $R G(t=2,768-p=0,0088)$, no pré-teste $(t=3,864-p=0,0004)$ e pós-teste $(t=7,264-p=$ 0,0000 ) do fator de RE. Os idosos, que no início do programa apresentavam-se mais sensíveis ao estresse que as idosas, conseguiram reverter a situação, conseguindo escores significativos no pós-teste $(t=5,025-p=0,0000)$ do fator de Interferência. Deste modo, conclui-se que os idosos conseguiram melhorar sua capacidade de reação ao estresse.

Palavras-chave: Idoso, Estresse, Defesa Pessoal.

\section{Abstract}

Innumerous factors affect the way that the elderly perform certain tasks, and these factors include health state, psychological characteristics, and the demands of the task. Even with the physical losses, some elderly people manage to overcome traumas and difficulties without suffering the negative consequences of stress. The elderly, aiming to lower their stress, practice tai chi chuan, Aqua Aerobics, Swimming, and other relaxing activities. However, little is known about personal defense as an alternative to reduce the stress in elderly people. This way, we carried out the present study, which had the aim of verifying in what way Self Defense contributes to the diminution of stress in the elderly. Then, 39 volunteers took part in the study (men=24, women=15) selected from the Center of Assistance of Physical Activities for the Elderly at the Free University of Brussels. The elderly participated for a period of 12 months, of a Personal Defense Course, adapted according to their physical characteristics. Jensen and Romer's Efficiency of Reaction to Stress Test, (1966) Stroop Color-Word Test - was applied to measure the attitudes of the subjects in situations of stress. The test is composed of General Speed Factors (GS), Specific Speed (SS) and Interference (INT), and it was applied at the beginning and the end of the course. The groups were similar in the pre-tests done at the beginning of the course, and statistically different at the end of the program. The men with higher scores, differentiated themselves statistically from the women in the pre-test of GS $(\mathrm{t}=2.768$ $-p=0.0088)$. Inversely, the women with a better emotional

Faculdade de Tecnologia e Ciências de Salvador - Salvador - Bahia - Brasil.

Revista de Educação Física 2008 Set; 142:58-65. Rio de Janeiro (RJ) - Brasil.

Recebido em 23.10.2007. Aceito em 03.07.2008. 
control, were outstanding in the pre-test $(\mathrm{t}=3.864-\mathrm{p}=$ $0.0004)$ and post-test of SS $(t=7.264-p=0.0000)$. This way, it is concluded that the men, who at the beginning of the course showed themselves more reactive to stress, after a regular practice of Personal Defense, managed to reach better results in the capacity of reaction to stress.

Key words: Elderly, Stress, Personal Defense.

\section{INTRODUÇÃO}

O estresse é uma alteração provocada quando o organismo tem que se adaptar a uma situação nova ou às mudanças que ocorrem, podendo ser, também, uma conseqüência das respostas físicas e mentais causadas pela ansiedade e pela depressão, em função da mudança brusca no estilo de vida do indivíduo. É, ainda, um fato natural e inevitável na vida e, para Delboni ${ }^{(2)}$, seja ele de natureza psicológica, física ou social, vem a ser um conjunto de reações fisiológicas que, conforme sua intensidade ou duração, pode levar a um desequilíbrio no organismo.

No meio esportivo, o estresse faz parte da vida do atleta, visto que a prática da atividade física, pela sua própria natureza, seus objetivos e suas características, tem o potencial de gerar estresse e ansiedade. A literatura documenta não só o potencial do estresse e da ansiedade no esporte ${ }^{(3)}$, mas, também, a diversidade e a interdependência dos fatores e processos psicológicos implicados no rendimento e no sucesso esportivo. Para Thomas ${ }^{(4)}$ e Fidelle ${ }^{(5)}$, o estresse e a ansiedade são capazes de contribuir negativamente no rendimento do atleta. Algumas modalidades, como as lutas e os esportes individuais, por exemplo, podem provocar níveis elevados de estresse. Um estudo, realizado por Cruz ${ }^{(6)}$, mostrou que essas variáveis são perturbações psicológicas capazes de influenciar o desempenho, estando presentes na maioria dos atletas, em situação pré-competitiva, competitiva e pós-competitiva, independentemente do sexo, da idade e do nível competitivo.

\section{Estresse no esporte}

Uma revisão da literatura mostra que a maioria das pesquisas investiga o estresse e a ansiedade de atletas de competição ${ }^{(7-9)}$. Com idosos, as pesquisas são mais direcionadas para analisar a qualidade de vida das pessoas idosas, praticantes de atividades físicas, enfatizando os efeitos benéficos para a saúde e para a motivação ${ }^{(10)}$, bem como para a prevenção das perdas das habilidades motoras ${ }^{(11,12)}$ e não locomotoras ${ }^{(13,14)}$. No campo psico-social, os estudos são direcionados para as perdas relacionadas à imagem corporal, à depressão, à auto-estima ${ }^{(15,16)}$ e à pouca motivação frente a realidade da vida ${ }^{(17)}$.

Para Chalvin (18) , os atletas são preparados para reagirem ao estresse, mas, entretanto, freqüentemente, enfrentam problemas com as pressões dos técnicos, dos dirigentes e da mídia, que fazem com que o atleta torne-se prisioneiro da sua própria imagem, passando a ter, no estresse, um fiel companheiro de treino e competições. Fidelle ${ }^{(5)}$ ressalta que o atleta idoso, assim como os jovens competidores, passa a ser confrontado com as cobranças de resultados feitas pelos treinadores, pelos dirigentes e pelos familiares. A conseqüência destas cobranças é estressante, podendo causar respostas psicológicas e fisiológicas, no idoso, capazes de deteriorar a sua qualidade de vida.

Tem-se observado, no entanto, nos últimos anos, que o esporte para idosos tem experimentado um crescimento importante, com suas formas de treino e disputa competitiva. Este crescimento coincide com o fenômeno mundial da longevidade, para o qual vários fatores vêm contribuindo, entre eles, a evolução da medicina no tratamento e no controle das doenças, as melhorias das condições sócio-econômicas e a preocupação com a adoção de um estilo de vida saudável.

Partindo destes tópicos iniciais, a presente pesquisa foi desenvolvida com o objetivo de verificar de que modo a Defesa Pessoal contribui para a diminuição, ou não, do estresse em idosos.

\section{METODOLOGIA}

\section{Amostra}

A pesquisa teve, como critério de inclusão, idosos com idade igual ou superior aos 60 anos, selecionados no Programa de Atividades Físicas do Conselho de Educação Permanente da Universidade Livre de Bruxelas (ULB). Devido a problemas de saúde, freqüência e limitação dos 
movimentos, 23 sujeitos foram excluídos da pesquisa. A amostra final foi composta por 39 sujeitos, sendo 24 homens, com média de idade de 65,3 $\pm 6,2$ anos, e 15 mulheres, com média de 62,6 $\pm 5,6$ anos.

Os indivíduos foram submetidos a uma avaliação médica que considerava características físicas, mentais, assim como histórico de enfermidades pregressas que impedissem a participação dos mesmos em atividades físicas. Para participar, voluntariamente, do curso de Defesa Pessoal, todos os indivíduos assinaram um Termo de Consentimento de Adesão e Participação, conforme as normas do Comitê de Ética em Pesquisa da ULB (CEP). O curso foi realizado no período de 12 meses, no Instituto Superior de Educação Física da ULB. Ao total, foram contabilizadas 96 aulas, com duração de 50 minutos cada sessão, sendo para tanto exigida a freqüência mínima de $75 \%$ dos idosos nas aulas teóricas e práticas.

Visando proteger a integridade física dos participantes, foram feitas algumas modificações no material usado nas aulas. Assim, as luvas e os protetores da cabeça e do tórax receberam um revestimento de espuma, sendo, também, os bastões confeccionados com material flexível. No início, os sujeitos receberam noções preliminares de Judô e aulas de Defesa Pessoal. As técnicas de ataque e defesa mais complexas foram eliminadas, sendo outras adaptadas em função da morfologia, da força e da constituição física dos indivíduos. Ao final de cada sessão, eram aplicadas técnicas de relaxamentos e um pouco de meditação, pois esta prática está relacionada ao relaxamento físico, à redução de estresse e à paz de espírito.

\section{Método}

Foi empregado, como instrumento, o Teste de Eficácia de Reação ao Estresse - Stroop Color-Word Test - de Jensen e Romer ${ }^{(1)}$, para medir as atitudes dos sujeitos em situações de estresse. O teste é constituído de três partes, que contempla informações referentes aos seguintes fatores:

- Rapidez Geral (RG), que tem como objetivo medir a velocidade dos sujeitos;

- Rapidez Específica (RE), que permite medir a reação à denominação das cores; e

- Interferência (INT), que é o resultado dos dois fatores (RG e RE), tendo como meta verificar a eficácia da resposta à reação ao estresse dos indivíduos testados.

O instrumento contempla informações de questões fechadas e abertas (recodificadas para múltipla escolha e múltiplas respostas), constituído das seguintes variáveis: idade, sexo, tipo de atividade física, tempo de prática e qual a atividade física praticada na juventude. As informações do Stroop Color são agrupas em três cartelas, tamanho A4, contendo retângulos dispostos em seis fileiras de quatro itens, impressos nas cores verde, rosa, azul e marrom, de modo que cada cor aparecesse de modo aleatório, apenas uma vez por fileira. A tarefa consiste em sublinhar as cores dos retângulos o mais rapidamente possível. No preenchimento de cores e palavras, a tarefa requer a modificação da atenção entre duas dimensões do estímulo (cor e palavra), implicando, desta forma, em uma concentração mais apurada, uma percepção e uma rapidez nas respostas. O levantamento dos dados é realizado, estabelecendo-se escores quanto ao número de categorias que o sujeito consegue completar corretamente, sendo os pontos atribuídos mediante o número de itens lidos, em cada parte, em um tempo estipulado de 45 segundos. A soma dos escores dos três fatores resulta em um percentual que classifica o grau de estresse dos sujeitos testados. Os idosos foram avaliados antes e no final do curso, sendo o instrumento aplicado em um ambiente neutro, contra estímulos visuais e sonoros, contando com a participação voluntária de pesquisadores do Laboratório de Psicologia do Esforço da ULB.

\section{Análise estatística}

O software Access foi utilizado na construção de um banco de dados para as questões contidas no instrumento de coleta. As informações foram transferidas para o pacote estatístico SPSS (Statiscal Package for Social Sciences).

$\mathrm{O}$ teste $\mathrm{t}$ de Student foi usado para comparar as amostras dependentes e independentes dos resultados intragrupos e intergrupos, pré e pós-intervenção. Foi, também, empregado o teste Kolmogorov-Smirnov, para verificar a normalidade da amostra, e o teste $F$ de Fischer-Snedecor, para as amostras independentes. Para todos os procedimentos, adotou-se um intervalo de confiança de $5 \%$, identificando-se, assim, o ponto das diferenças reveladas.

\section{RESULTADOS}

O curso teve como meta oferecer um programa de Defesa Pessoal, administrado de forma suave, visando o condicionamento físico, a postura, a autoconfiança e o 
FIGURA 1

AULA PRÁTICA DE INICIAÇÃO AO JUDÔ.

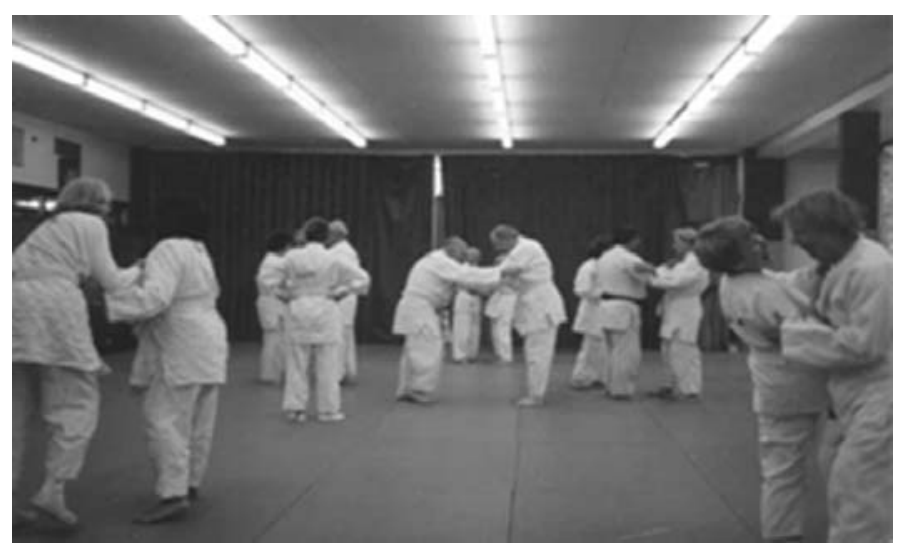

equilíbrio emocional dos idosos. Outra meta do programa era identificar os pontos mais frágeis do adversário, para atacar e defender-se dos golpes. Entretanto, no início do curso, $52 \%$ dos idosos tiveram dificuldades para assimilar as técnicas mais complexas de ataque e defesa. Esta dificuldade deve-se, em parte, às características físicas e técnicas dos indivíduos, que tiveram que melhorar, nos dois primeiros meses de atividades, a postura, a flexibilidade, a habilidade e, principalmente, a coordenação motora.

As mulheres, que tiveram mais dificuldades na assimilação das técnicas, receberam aulas suplementares de judô e sobre métodos especiais de autodefesa, adaptados às suas características físicas (FIGURAS 1 e 2). Os movimentos foram desenvolvidos, planejados e concentrados no aprimoramento de seus desempenhos, através de uma série de exercícios repetitivos de ataque e defesa. O objetivo era praticar Defesa Pessoal, e não fazer com que "rolasse, brigasse ou atacasse" seus companheiros, mas que despertasse o conhecimento dos pontos mais frágeis do adversário, para aplicar e saber como se defender dos golpes ${ }^{(19,20)}$. Os resultados foram elucidativos, pois a Defesa Pessoal mostra dimensões de percepções de ameaça e de ausência de controle, em uma atividade que requer concentração e precisão nos movimentos.

De acordo com Shumway e Woollacott ${ }^{(21)}$, as pessoas, à medida em que envelhecem, passam de uma forma ativa de controle para uma forma passiva de lidar com eventos. Uma das desvantagens sofridas pelos idosos é o fato de que, progressivamente, vão deixando de receber estímulos, tendo menos oportunidades para viverem situações
FIGURA 2

TÉCNICAS DE EQUILÍBRIO E ROLAMENTO.

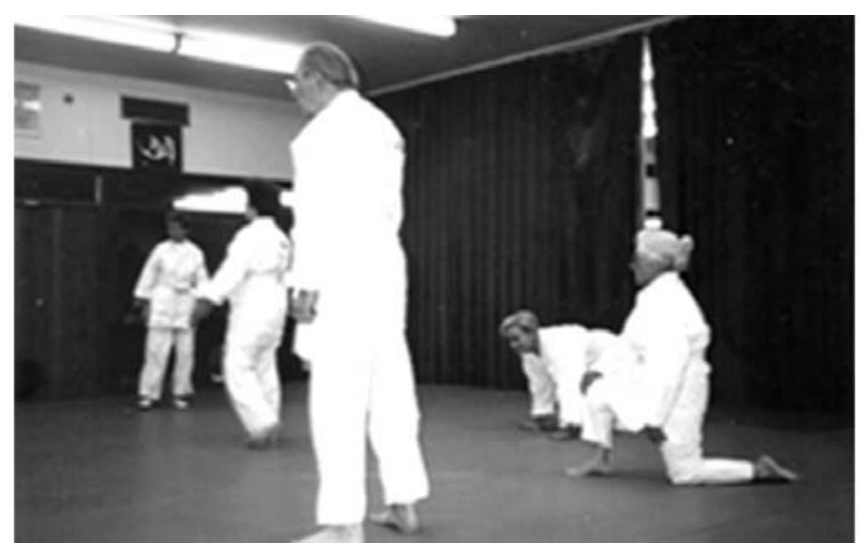

distintas, o que dificulta a capacidade de adaptação a novas situações e novas aprendizagens.

\section{Teste de Rapidez Geral (RG)}

Uma primeira área de investigação mostrou que os idosos participavam do curso de Defesa Pessoal para satisfazer as necessidades fundamentais, como a competência e a autodeterminação. Essa satisfação pessoal pode ser caracterizada por uma motivação intrínseca, que, de acordo com a teoria da avaliação cognitiva de Deci e Ryan (22), o indivíduo sente, primeiramente, necessidade de melhorar ou de demonstrar sua eficácia, e, depois, ele procura tomar decisões e exercer um controle sobre suas atividades.

A análise dos dados coletados mostrou a existência de resultados estatisticamente significativos entre os sujeitos dos dois grupos. Assim, constatou-se que, no fator de Rapidez Geral, as idosas obtiveram, no início do curso, escores elevados no pré-teste, diferenciando-se inclusive dos idosos ( $t=2,768-p=0,0088)$. Entretanto, no pós-teste, elas não conseguiram o mesmo resultado obtido no pós-teste pelos idosos, permanecendo com os mesmos escores nas duas avaliações realizadas (TABELA 1).

Os 24 idosos, do sexo masculino, ao final do curso, conseguiram melhorar o nível de RG, estabelecendo, para tanto, a média de 3,738 pontos, uma diferença estatisticamente significativa em relação às idosas $(t=5,812-p=0,0000)$.

Dentre os eventos de vida estressantes que compartilham no dia-a-dia, reverter a situação foi, para os idosos, o resultado de suas estratégias de enfrentamento, 
TABELA 1

MÉDIA DOS ESCORES NO TESTE DE RAPIDEZ GERAL (RG).

\begin{tabular}{|c|c|c|c|c|c|}
\hline Teste & Sexo & Média & $\begin{array}{l}\text { Desvio } \\
\text { Padrão }\end{array}$ & $\mathrm{t}$ Student & $\mathrm{p}=$ \\
\hline \multirow[t]{2}{*}{ Pré-teste } & $\mathrm{h}=24$ & 1,796 & 0,3483 & 2,768 & $0,0088^{*}$ \\
\hline & $m=15$ & 2,447 & 1,072 & & \\
\hline \multirow[t]{2}{*}{ Pós-teste } & $h=24$ & 3,738 & 0,8596 & 5,812 & $0,0000^{*}$ \\
\hline & $m=15$ & 2,113 & 0,8314 & & \\
\hline
\end{tabular}

${ }^{*} \mathrm{p}<0,05=$ resultado significativo $-\mathrm{NS}=$ não significativo

além do interesse em participar de todas as atividades técnicas no transcorrer do curso. Este resultado é ainda expresso nos valores médios, obtidos por esses sujeitos, nos dois testes, com uma variação de 1,796 a 3,738 pontos (TABELA 1). Assim sendo, a motivação dos pesquisados, bem como o seu interesse, parecem exercer uma forte influência no controle do seu estresse.

Algumas idosas, mesmo recebendo aulas suplementares, revelaram, no transcorrer do curso, as dificuldades encontradas, assim como um temor em praticar uma arte marcial pela primeira vez na vida (FIGURA 3). Elas não tinham confiança no que realizavam, estando menos preocupadas com o que não iriam fazer do que com o que iriam fazer. Além disso, pensavam que o ataque poderia resultar em fracasso, o que deixava parte do grupo fora de seu controle.

Para Samulski ${ }^{23)}$, a autoconfiança é importante, tanto para amadores, quanto para profissionais, e, para obter rendimento em qualquer atividade física, o indivíduo tem

FIGURA 3

TÉCNICAS DE ARTICULAÇÕES DO BRAÇO.

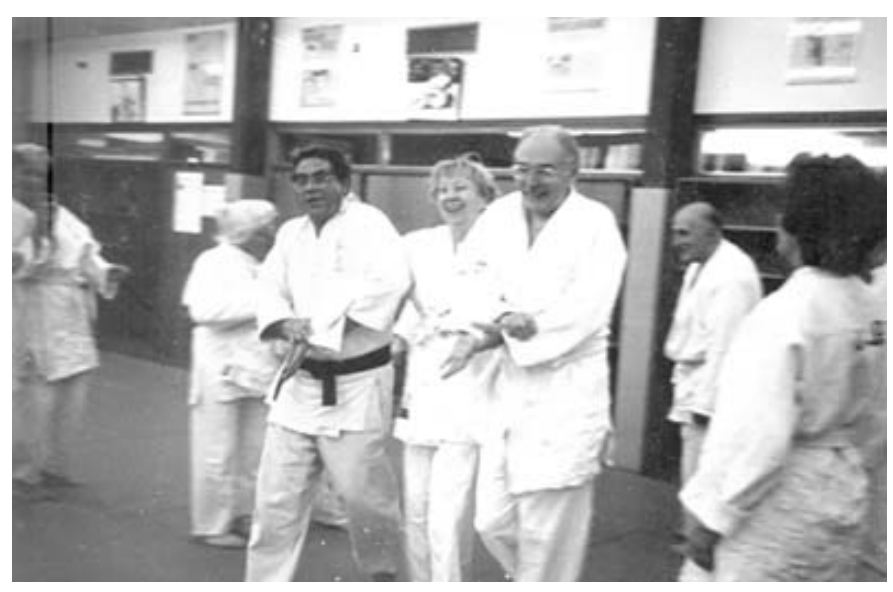

que revelar um maior senso de confiança no seu talento e nas suas habilidades. Garratt ${ }^{(24)}$ cita que o medo de fracassar jamais deve ser subestimado, mesmo no caso de esportistas ocasionais. Em muitas situações, cria-se um estresse adicional, tanto pelo medo de fracassar, ou de parecer tolo, quanto por tentar algo e ter de enfrentar as conseqüências.

Segundo Cruz ${ }^{(6)}$, tradicionalmente, o estresse e a ansiedade no meio esportivo têm sido vistos como fatores perturbadores que, invariavelmente, prejudicam o rendimento dos atletas, constituindo-se um problema usual e preocupante para os que lidam, direta ou indiretamente, com o esporte. Dados de diversos estudos evidenciam a elevada incidência de estresse e de ansiedade em contextos esportivos, experimentados por muitos atletas, independente da idade, do tempo de prática, da experiência competitiva ou de aspectos climáticos e ambientais $(25,26)$.

\section{Teste de Rapidez Específica (RE)}

O teste de Rapidez Especifica mede a reação à denominação das cores, requerendo, desta forma, uma concentração mais apurada, além de percepção e de rapidez nas respostas dos indivíduos testados. Esta percepção e rapidez das respostas às cores contidas, no teste de Jesen e Romer (1), foram mais evidentes nos resultados das idosas no pré e pós-teste. A média obtida pelas idosas, nas duas avaliações, realizadas no início e no final do curso, foram excelentes, com escores que oscilavam entre 3,140 a 3,573 pontos (TABELA 2). Esta pontuação fez com que, no confronto com os idosos, elas mostrassem diferenças estatisticamente significativas no pré-teste $(t=3,864-p=0,0004)$ e no pós-teste $(t=7,264$ $p=0,0000$ ), resultando, para elas, em múltiplas estratégias de enfrentamento e um excelente controle emocional.

Para Jensen e Romer (1), as mulheres apresentam, sempre, uma superioridade no teste de Rapidez Específica, 
TABELA 2

MÉDIA E DESVIO PADRÃO DO FATOR DE RAPIDEZ ESPECÍFICA.

\begin{tabular}{lccccc}
\hline Teste & Sexo & Média & D.Padrão & t Student & p= \\
\hline Pré-teste & $\mathrm{h}=24$ & 2,104 & 0,5879 & 3,864 & $0,0004^{\star}$ \\
Pós-teste & $\mathrm{m}=15$ & 3,140 & 1,089 & & $0,0000^{*}$ \\
& $\mathrm{~h}=24$ & 1,983 & 0,6742 & 7,264 & 0,6497 \\
\hline
\end{tabular}

${ }^{*} \mathrm{p}<0,05=$ resultado significativo $-\mathrm{NS}=$ não significativo

se comparadas aos homens, justificando, portanto, os escores obtidos pelas idosas nos dois testes. Este mesmo resultado foi observado por Guedes ${ }^{(27)}$, em pesquisas realizadas com idosos belgas, dos dois sexos, praticantes de ginástica e natação.

Outro ponto importante observado, é que, para suprir sua pequena capacidade técnica e a pouca experiência no meio esportivo, as idosas, através dos treinos adicionais e das técnicas de relaxamento e de concentração, conseguiram se superar e manter a média no pós-teste, motivadas, em parte, pela sua recuperação nas aulas de Defesa Pessoal. Para elas, no momento do ataque e da defesa, a concentração é crucial, não podendo ser conseguida pelo simples desejo, pois necessita de um engajamento no que se faz naquele momento particular. Este é um estado difícil de alcançar, principalmente no tatame, local onde duas pessoas, nas figuras de atacante e atacado, parecem fundir-se em uma só. Leonard ${ }^{(28)}$, inclusive, cita que as artes marciais apresentam uma mistificação do ataque amoroso e o da reconciliação pacífica.

\section{Teste de Interferência (INT)}

O fator de Interferência procurou medir a capacidade de reação ao estresse dos indivíduos participantes da pesquisa, associando, para tanto, os resultados estatísticos conseguidos pelos idosos nos fatores de Rapidez Geral e Rapidez Específica.
Constatou-se, na comparação intragrupos, um melhor resultado dos idosos no pré e no pós-teste ( $\mathrm{t}=4,964$ $p=0,0000)$. As idosas permaneceram com as médias baixas, nas duas avaliações (TABELA 3).

Com relação à comparação entre gêneros, observou-se que não houve diferenças significativas no pré-teste (TABELA 3). Entretanto, no pós-teste, os idosos souberam melhor dosar os eventos estressantes, conseguindo diferenças estatisticamente significativas em relação ao grupo das idosas $(t=5,025-p=0,0000)$.

Diversos fatores contribuíram para um melhor resultado dos idosos em relação às idosas, dentre estes a sua maior experiência e vivência esportiva. Neste sentido, constatou-se que a maior parte dos homens idosos pesquisados já havia praticado Defesa Pessoal (18\%) ou outras atividades esportivas, como por exemplo a natação (36\%), o ciclismo (36\%) e o futebol (72\%), sendo, portanto, natural que as estratégias de enfrentamento fossem mais administradas por eles, além desta vivência esportiva facilitar um maior domínio das técnicas de autodefesa.

Por outro lado, o estudo mostrou que $92 \%$ das idosas nunca haviam praticado atividade esportiva, sendo, portanto, normal seu pouco desempenho técnico.

Para Thomas ${ }^{(4)}$, grande parte dos estudos realizados neste domínio mostra uma diminuição da eficácia da reação ao estresse, que aumenta com a elevação da idade.

TABELA 3

MÉDIA DOS ESCORES NO TESTE DE INTERFERÊNCIA (INT).

\begin{tabular}{|c|c|c|c|c|c|}
\hline Teste & Sexo & Média & D.Padrão & t Student & $p=$ \\
\hline \multirow[t]{2}{*}{ Pré-teste } & $\mathrm{h}=24$ & 2,375 & 0,9166 & 1,901 & $0,0651-N S$ \\
\hline & $m=15$ & 1,880 & 0,5226 & & \\
\hline \multirow[t]{2}{*}{ Pós-teste } & $\mathrm{h}=24$ & 3,767 & 1,014 & 5,025 & $0,0000^{*}$ \\
\hline & $m=15$ & 2,333 & 0,5447 & & \\
\hline
\end{tabular}

${ }^{*} \mathrm{p}<0,05=$ resultado significativo $-\mathrm{NS}=$ não significativo. 
No entanto, não encontramos esta correlação entre os sujeitos do sexo masculino. Os efeitos do estresse, no idoso, são individualizados, em função da personalidade, das características e das exigências de cada situação. Segundo Nahas ${ }^{(29)}$, as características do indivíduo e dos agentes estressantes é que determinarão as formas mais apropriadas para lidar com o estresse. Existem diferentes respostas a situações de estresse, dependendo de fatores como: hereditariedade, idade, sexo, estilo de vida, estado de saúde, experiências anteriores e tipo de personalidade. Para Chopra ${ }^{(30)}$, os corpos que envelhecem simplesmente parecem se desgastar como máquinas. Entretanto, diferentemente das máquinas, que se desgastam com muito uso, o corpo humano é capaz de ficar bem melhor se for usado. Um músculo bem exercitado não deteriora, ao contrário, fica mais forte.

\section{CONCLUSÃO}

A presente pesquisa teve como objetivo mostrar que a Defesa Pessoal, mesmo sendo uma atividade que requer concentração e precisão de movimentos, pode ser praticada por indivíduos com idade avançada, sem o risco de provocar danos físicos ou psicológicos aos seus integrantes. O esforço físico, para pessoas com idade avançada, é uma questão de dosagem e, para indivívuos idosos com uma saúde razoável, os conhecimentos de alguns princípios de prevenção e de autodefesa podem melhorar a vida.
Quanto ao resultado do Stroop Color Test, o estudo mostrou que as idosas responderam com maior precisão ao fator de Rapidez Específica, no início e no final do curso, diferenciando-se estatisticamente dos idosos. Entretanto, não souberam como administrar seu controle emocional, bem como suas estratégias, frente aos agentes estressantes no transcorrer do curso de autodefesa. Cabe ressaltar que essa dificuldade deve-se, em parte, à pouca vivência e experiência esportiva das mesmas.

A Defesa Pessoal provocou mudanças físicas e estressantes, capazes de produzir alterações comportamentais e necessidade de adaptação. Estas mudanças não atingiram os idosos, que permaneceram estáveis e com um maior controle e domínio dos eventos. Isto pode ser explicado pela maior maturidade esportiva do grupo masculino, suas características físicas, além de um melhor controle emocional, sendo estes os fatores capazes de promover as diferenças entre os gêneros.

Concluiu-se que a metodologia utilizada foi adequada para confirmar que a Defesa Pessoal é uma atividade eficaz para melhorar o estresse de indivíduos de idade avançada. Sugere-se que mais estudos sejam realizados, com um grupo maior de pesquisados, na perspectiva de se verificar e identificar os benefícios da Defesa Pessoal como meio de reduzir o estresse de pessoas idosas, tendo como base variáveis como, por exemplo, idade, sexo, estilo de vida, estado de saúde, características socioeconômicas e outros eventos estressantes.

\section{REFERÊNCIAS BIBLIOGRÁFICAS}

1. Jensen AR, Romer WD. The stroop color word test. London, England: Review Acta Psychologica, 1966; 25(1): 36-93.

2. Delboni TH. Vencendo o stress. São Paulo: Makron Books; 1997.

3. Scanff C. Stress et sport, la gestion du estresse. Revue EPS 1994:22-4.

4. Thomas R. Prépartion psychologique du sportif. Paris: Vigot; 1991.

5. Fidelle G. Estresse et sport, étude chez le sportif de haut niveau. Revue EPS 1994; 247: 21-2.

6. Cruz JF. Estresse e ansiedade na competição desportiva: natureza, efeitos e avaliação. Braga: Lusografe; 1996:173-214.

7. Vanfraechen-Raway R. Éthodologique appliquée avec des participants da trosième âgée. Revue Sport Santé 1991; $152: 9-15$.

8. Kroll W. Le estresse chez les atlètes de haut niveau, coach athlete and the sport psychologist. INSEP 1979; 549: 211-9.

9. Pfister R. Prévenir le estresse du sportif de haut niveau. Le Journal des Psychologues 1987;49: 28-30.

10. Vallerand RJ, Thill E. Introduction à la psychologie de la motivation. Paris, France: Editions Vigot, 1993. 
11. Niellens H. Béneficies de la pratique régulière d 'Activités phyisiques sur l’appareil locomoteur. Revue Sport 1998; 163: $13-4$.

12. Toulotte CA, Dangremont B, Fabre C. Equilibre et entraînement des seniors chuteurs. In: Proceedings, Egrepa - 8th International Congress. Bélgica: Universidade Livre de Bruxelas; 2000: 293-6.

13. Vrijens J. L' Entraînement raisionné du sportif. Bruxelles: Editions de Boeck-Wesmael; 1991.

14. Weiss U. Les personnes âgées et le sport. Revue de l'Ecole Fédérale de Gymynastique et de Sport 1989; 9-11.

15. Hétu JL. Psychologie du Vieillissement. Canadá: Méridien; 1992.

16. Rillaer VJ. La gestion de soi. Liège: Mardaga; 1992.

17. Lucena RMG. Approche des caractéristiques psychologiques des personnes âgées pratiquant des activités sportives [Thèse de Master en Éducation Physique]. Bruxelles: Université Libre de Bruxelles; 1993.

18. Chalvin D. Faire face aux stress dans le quotidianne. Paris: Editions ESF; 1982.

19. Habersetzer R. Self-défense: techniques de défense à mais nue pour tous. Paris: Amphora; 1990.

20. Ortega JN. Self-defense: comportements face à l'agression. Paris: Éditions Albin Michel; 1991.

21. Shumway CA, Woollacott MH. Controle motor: teoria e aplicações práticas. Barueri, SP: Manole; 2003

22. Deci EL, Ryan RM. Intrinsic motivation and self-determination. New York, USA: Plenum; 1985.

23. Samulski DM. Psicologia do esporte: manual para educação psicologica e fisioterapia. São Paulo, SP: Manole; 2002.

24. Garratt T. Motivação esportiva: aumentando o desempenho nos esportes. São Paulo, SP: Madras; 1999.

25. Guedes OC. Efficacité de la reaction au stress chez des personnes agêes. Poitiers: Congrès International de la Société Française de Psychologie du Sport; 1994: 45-8.

26. Guedes OC. La pratique de la self-defense comme moyen d'améliorer le estresse des personnes agées. In: Proceedings, Egrepa - 8th International Congress. Bélgica: Universidade Livre de Bruxelas; 2000: 293-296.

27. Guedes OC. Intruduction aux fondements théoriques et pratiques de la Self-Défense. Bruxelles: Editions ULB; 1992.

28. Leonard G. O atleta dos atletas: uma nova dimensão para a atividade Física. São Paulo, SP: Summus; 1999.

29. Nahas MV. Atividade fisica, saúde e qualidade de vida : conceitos e sugestões para um estilo de vida ativo. Londrina, Paraná: Midiograf; 2006.

30. Chopra D. Corpo sem idade, mente sem fronteiras. 8ª Ed. São Paulo, SP: Rocca; 2002.

\section{Endereço para correspondência:}

Onacir Carneiro Guedes Rua Emilio de Araújo Chaves, 167 - Altiplano João Pessoa - Paraíba - Brasil CEP 58056-150

Tel.: (83) 3252-1485 e-mail: onacirguedes@ig.com.br 